\title{
A Dual Tuned Complementary Structure Frequency Selective Surface for WLAN Applications
}

\author{
Arup Ray, Manisha Kahar, Sushanta Biswas, Debasree Sarkar, Partha Pratim Sarkar \\ DETS, University of Kalyani ,741235,India \\ email:iamarupray@gmail.com,manishakahar@gmail.com,biswas.su@gmail.com, \\ dsarkar70@gmail.com,parthabe91@yahoo.co.in
}

\begin{abstract}
A single substrate layer double metallic plane frequency selective surface (FSS) is designed with miniaturized electrical elements and two tunable pass-bands enabling Wireless Local Area Network applications. Each FSS periodic cell consists of a metallic square loop on the top metal layer and its complementary etched on the bottom metal layer separated by a thin dielectric layer. This designed FSS provides two tunable transmission bands. For predicting the frequency selective characteristics of the designed FSS an equivalent electric circuit model is also presented. To satisfy the needed requirements for Wireless Local Area Network (WLAN) applications the designed structure is optimized. Due to symmetrical nature of the design, the FSS is insensitive to variations of $\mathrm{RF}$ incidence angle for $90^{\circ}$ (degree) rotations of the FSS. Simulated theoretical investigations are done by Ansoft Designer® software. Experimental investigation is performed using standard microwave test bench.
\end{abstract}

Index Terms - Complementary Structure, Frequency Selective Surface, Multi-frequency operation, Polarization independent, WLAN

\section{INTRODUCTION}

Frequency-selective surfaces (FSS) are formed by a two-dimensional array of metallic patterns printed on a dielectric substrate [1]-[2]. FSS are of two categories: patch array and aperture array, and they exhibit stop-band characteristic and pass-band characteristic respectively. Thus FSSs have been widely used as spatial filters to reflect totally or transmit electromagnetic waves in some specified frequency bands. Accordingly, these FSSs can be applied in radome, filter, dichroic subreflector, directivity enhancement, and radar cross section (RCS) reduction. [3]-[6].In this paper, we have achieved a novel FSS structure on a double metallic-layer polytetrafluoroethylene (PTFE),substrate having two metallic planes with dual [7]-[11] pass bands for WLAN application, at $2.4 \mathrm{GHz}$ and $5.2 \mathrm{GHz}$

IEEE 802.11b Wireless Local Area Network (WLAN) [12] standards use the same unregulated radio signaling frequency, $2.4 \mathrm{GHz}$ as the original 802.11 standard. Vendors often prefer using these frequencies to lower their production costs. Being unregulated, $802.11 \mathrm{~b}$ gear can incur interference from microwave ovens, cordless phones, and other appliances using the same $2.4 \mathrm{GHz}$ range. However, by 
installing $802.11 \mathrm{~b}$ gear at a reasonable distance from other appliances, interference can easily be avoided.

802.11a WLAN standards [13] support bandwidth up to $54 \mathrm{Mbps}$ and signal in a regulated frequency spectrum around $5 \mathrm{GHz}$. This higher frequency compared to $802.11 \mathrm{~b}$ shortens the range of 802.11a networks. The higher frequency also means 802.11a signals have more difficulty penetrating walls and other obstructions. Because $802.11 \mathrm{a}$ and $802.11 \mathrm{~b}$ utilize different frequencies, the two technologies are incompatible with each other however some vendors offer hybrid 802.11a/b network gear.

The geometry of the designed FSS element in this paper is a metallic square loop on the top metal plane together with its complementary etched on the bottom plane This first designed FSS however did not meet the requirements of the aforesaid WLAN so it was optimized by meandering the metallic portions of the square loop on all sides. The equivalent circuit model of the FSS is also explained.

We have analyzed the proposed Frequency Selective Surface structure theoretically by Ansoft Designer ${ }^{\circledR}$ software which works on the Method of Moments. The simulated results obtained have been compared with experimental measured data. It is interesting to note that the simulated theoretical and experimental results are in close agreement.

\section{DESIGN OF THE FREQUENCY SELECTIVE SURFACE}

Our proposed FSS uses two metallic planes separated by a thin dielectric substrate. Each square loop patch on the top metal surface of the FSS structure has the outer most dimensions of $20 \mathrm{~mm} \times 20$ $\mathrm{mm}$ and a uniform metallic loop width of $5 \mathrm{~mm}$ as shown in Fig. 1. The design complementary to the above is etched on the bottom metal plane shown in Fig 2. and the 3-D view of a single cell of the complete FSS is shown in Fig. 3 .The distance between the centers of any two adjacent square loops is $21 \mathrm{~mm}$ in both the horizontal and vertical directions respectively. Spacing between two consecutive patches in a row or a column is maintained in such a way that the rule governing a conventional array antenna is maintained. A general rule is that the spacing between two adjacent patches should be less than one wavelength for the broad side incident cone, i.e. $0^{\circ}$ incident angles [14]. For our case the spacing is $2 \mathrm{~mm}$ between any two adjacent elements which is less than one wavelength. It is interesting to note that for a square loop resonating element, wavelength corresponding to resonant frequency is equal to the perimeter of the resonating element [15]. Dimension of the polytetrafluoroethylene (PTFE) substrate are $150 \mathrm{~mm} \times 150 \mathrm{~mm} \times 0.79 \mathrm{~mm}$. The relative dielectric constant is 2.4. As the dielectric structure is very thin, the structure was not affected by loading effect [15]. The square loop element is chosen as it is quite insensitive to polarization changes, being symmetric about any orthogonal co-ordinate system and has a natural broad bandwidth. 


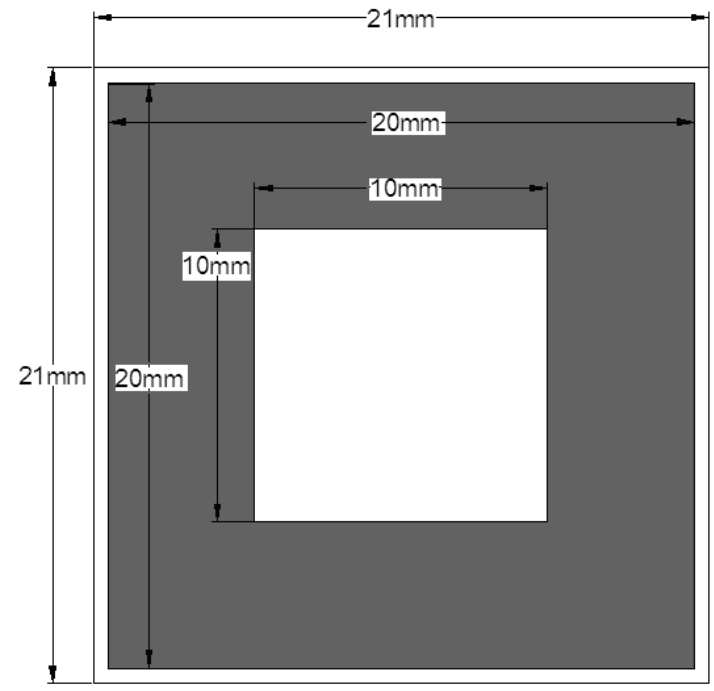

Fig 1 Top view of the proposed unit cell (black portions indicate copper)

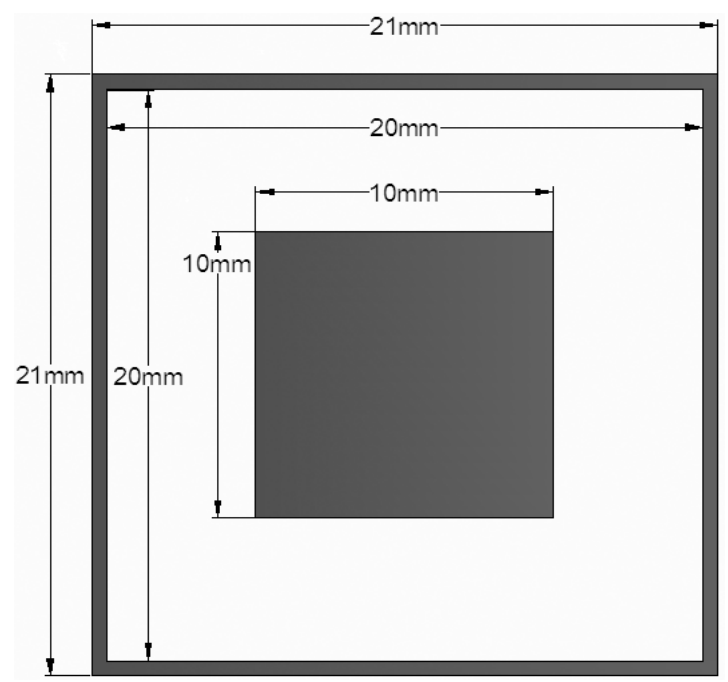

Fig 2 Rear view of the proposed unit cell (black portions indicate copper)

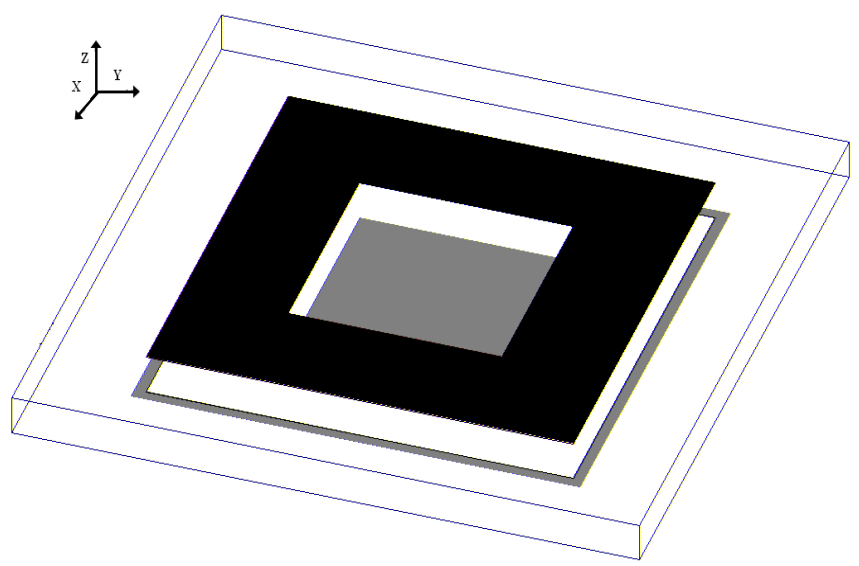

Fig 3 3D overview of single cell of the complete FSS (shaded regions indicate copper)

According to the Babinet's principle in electro-magnetics [15]-[16], a complementary structure is supposed to have exactly the complementary response to the original one. A metallic loop strip 
contributes to inductance while the gap between two elements provides capacitance [17]. Thus for a complementary part, the original band reject characteristics will be replaced by a bandpass nature. A metallic square loop FSS (shown in Fig. 1), which is equivalent to a series LC resonant circuit, providing a transmission zero ,its complementary structure is a slot square loop (shown in Fig. 2), which is equivalent to a parallel LC resonant circuit, thus providing a transmission pole. This is verified from the simulated plot shown below in Fig. 4 obtained using Ansoft Designer ${ }^{\circledR}$ software.

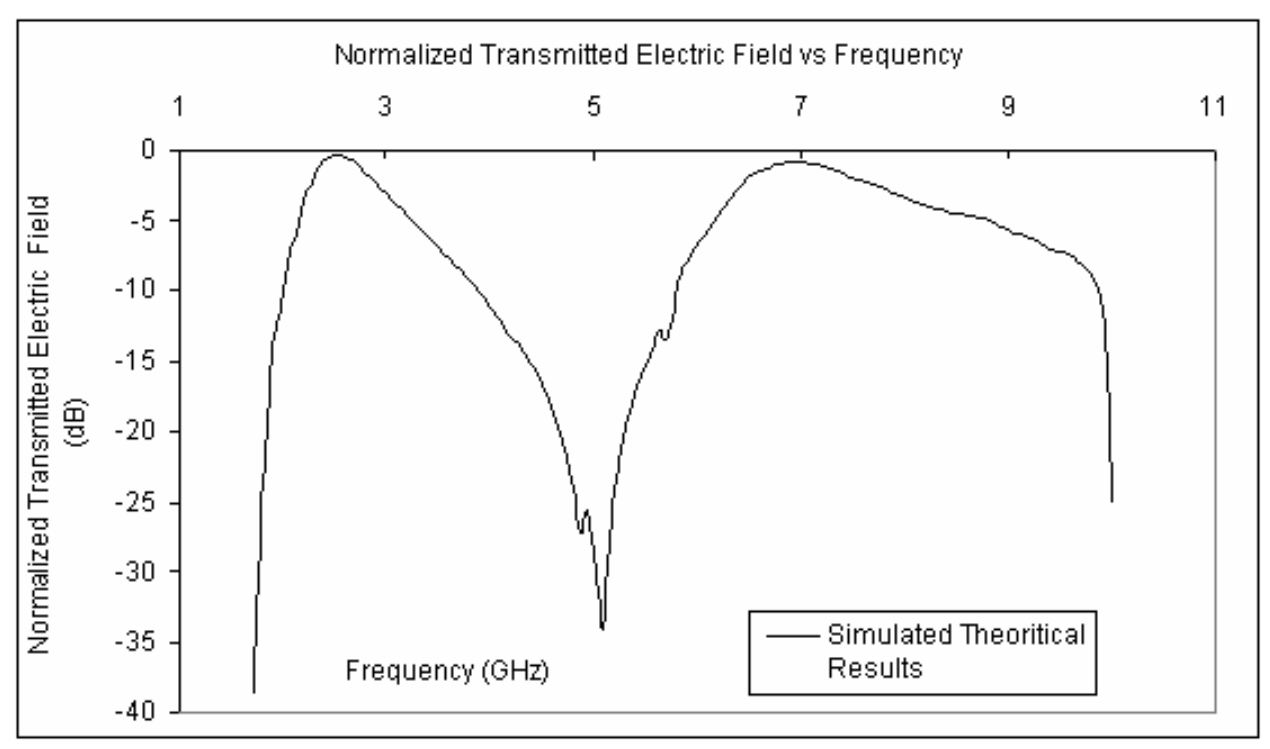

Fig 4. Normalized transmitted electric field vs frequency for FSS shown in Fig 3.

However the frequency response (shown in Fig. 4) of the above designed FSS was not satisfying the needs of WLAN. From Fig. 4 it is clearly visible that we obtain two resonant frequencies one close to $3 \mathrm{GHz}$ and the other at about $7 \mathrm{GHz}$. This means that the structure requires miniaturization, in order to tune it to lower frequencies. Miniaturization is generally done by two process viz. by using dielectric with high permittivity or by increasing the effective electrical length of the metallic patch. The former method is generally avoided because of their greater losses; they are less efficient and have relatively smaller bandwidths [18]. So the second method of miniaturization is a better choice which is done by meandering of the excited metal patch surface current or by embedding suitable slots on the excited metal patch.

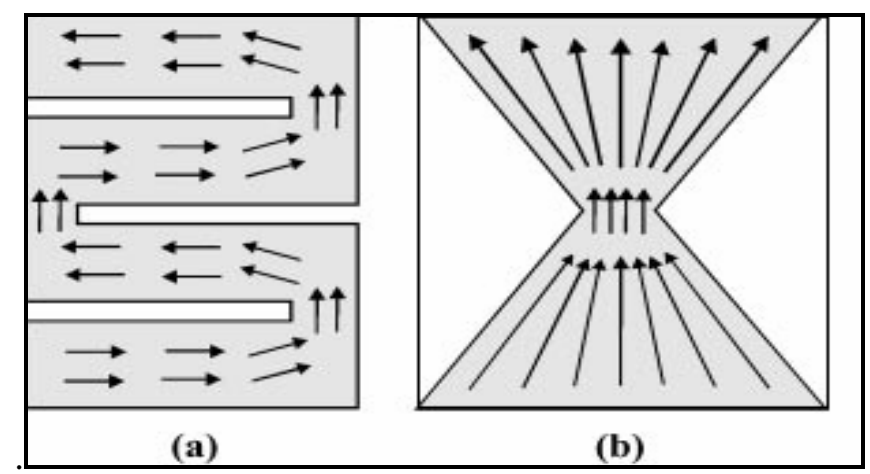

Fig 5. Increasing the effective electrical length of the metallic patch (shaded portions indicate metal while arrow marks the current): (a) meandering of the excited metal patch surface current (b) embedding suitable slots on the excited metal patch. 
When a plane-wave illuminates an array of metallic elements, it excites electric current on the elements. The amplitude of the generated current depends on the strength of the coupling of energy between the wave and the elements. The coupling reaches its highest level at the fundamental frequency. As a result, the elements are shaped so that they are resonant near the frequency of operation. Depending on element shape and distribution, the current itself acts as an electromagnetic source, thus producing a scattered electric field. The scattered field added to the incident field constitutes the total field in the space surrounding the FSS. By controlling the scattered field (i.e is by properly shaping the designed resonant elements), the required filter response is produced.

Accordingly we modified our design by introducing eight rectangular slots of dimensions $2 \mathrm{~mm} x$ $1 \mathrm{~mm}$ (optimized length after rigorous parametric study) mounted on the inner dimension of metal square loop on the top metallic layer of FSS and its complementary etched design on the bottom metallic layer of FSS shown respectively in Fig. 6 and Fig. 7 while the 3-D view of a single cell of the complete FSS is shown in Fig. 8.This is the newly designed optimized FSS structure.The protype of the designed FSS is shown in Fig. 9 (top view) and Fig. 10 (Rear view).

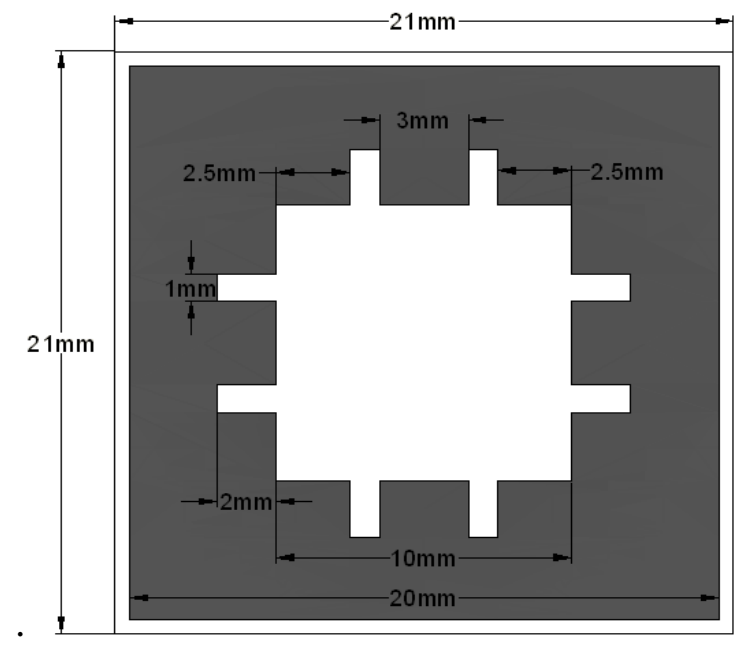

Fig 6 Top view of the modified meandered unit cell (black portions indicate copper)

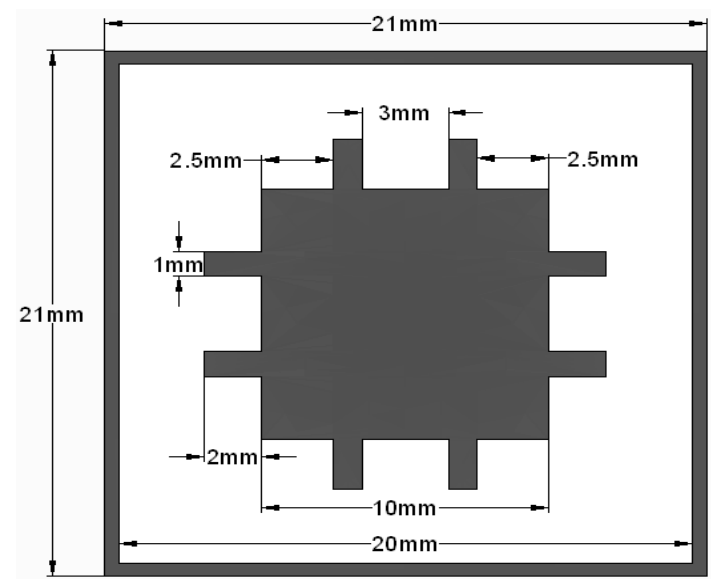

Fig 7 Rear view of the modified meandered unit cell (black portions indicate copper) 


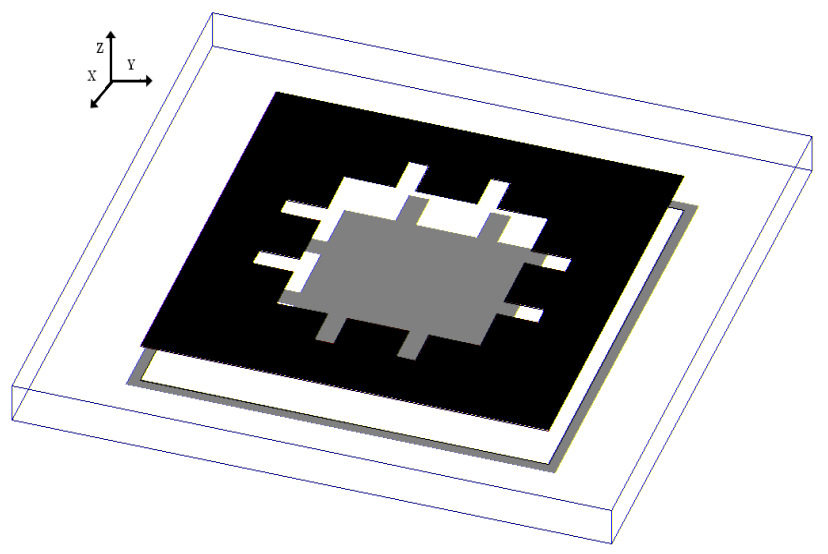

Fig 8. 3D overview of the modified meandered unit cell (shaded regions indicate copper)

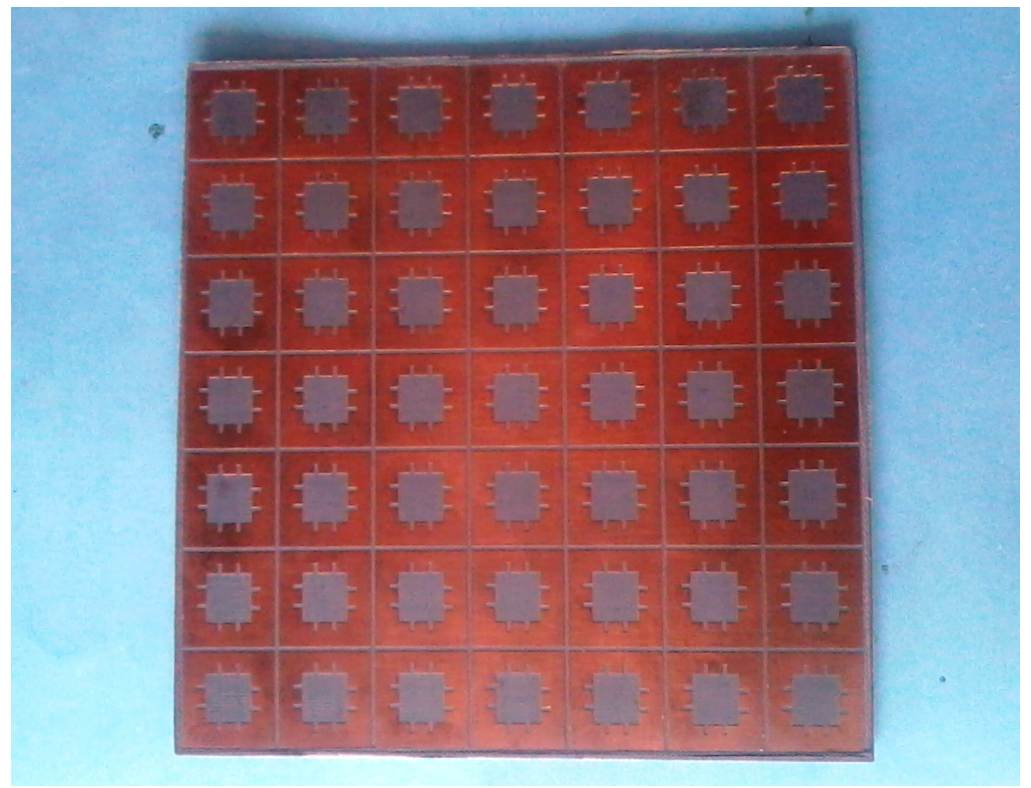

Fig 9.Top view of the protype designed FSS

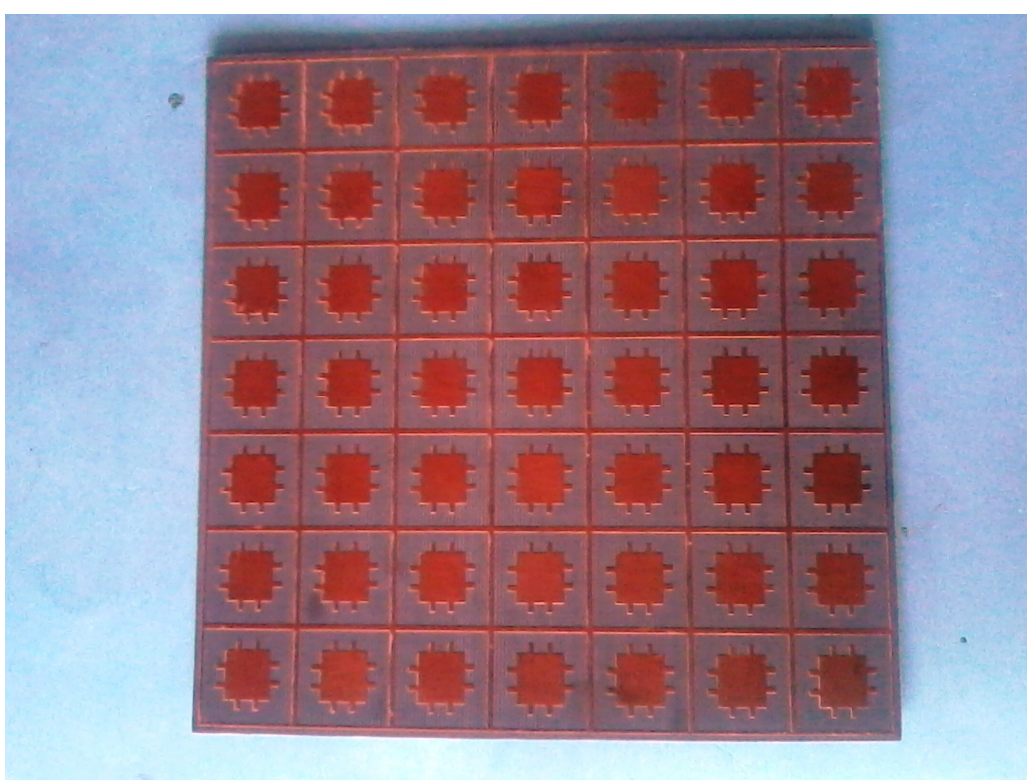

Fig 10. Rear view of the protype designed FSS 


\section{RESULTS AND DISCUSSION}

Transmission test for the FSS structure has been performed using standard microwave test bench, shown in Fig. 11 .The transmitting horn antenna is connected to a variable R.F oscillator (Marconi Microwave Source, Model No: 6058B) and the receving horn antenna to a Agilent Power meter (Model No. Agilent E4418B) with Power sensor (Model No. Agilent E4412A). The proposed FSS is kept on a wooden frame stand at far field distance of the transmitting horn antenna. The R.F oscillator radiates power which is blocked at all frequencies except at $2.4 \mathrm{GHz}$ and $5.2 \mathrm{GHz}$. This is indicated from the readings of the power meter in $\mathrm{dBm}$. These values were normalized and a graph of measured normalized transmitted electric fields vs. frequency for the FSS structure, shown in Fig. 8, was obtained as shown in Fig. 12. On the same graph we have plotted the simulated results for the FSS structure shown in Fig. 8, using Ansoft Designer® software for convenient comparison.

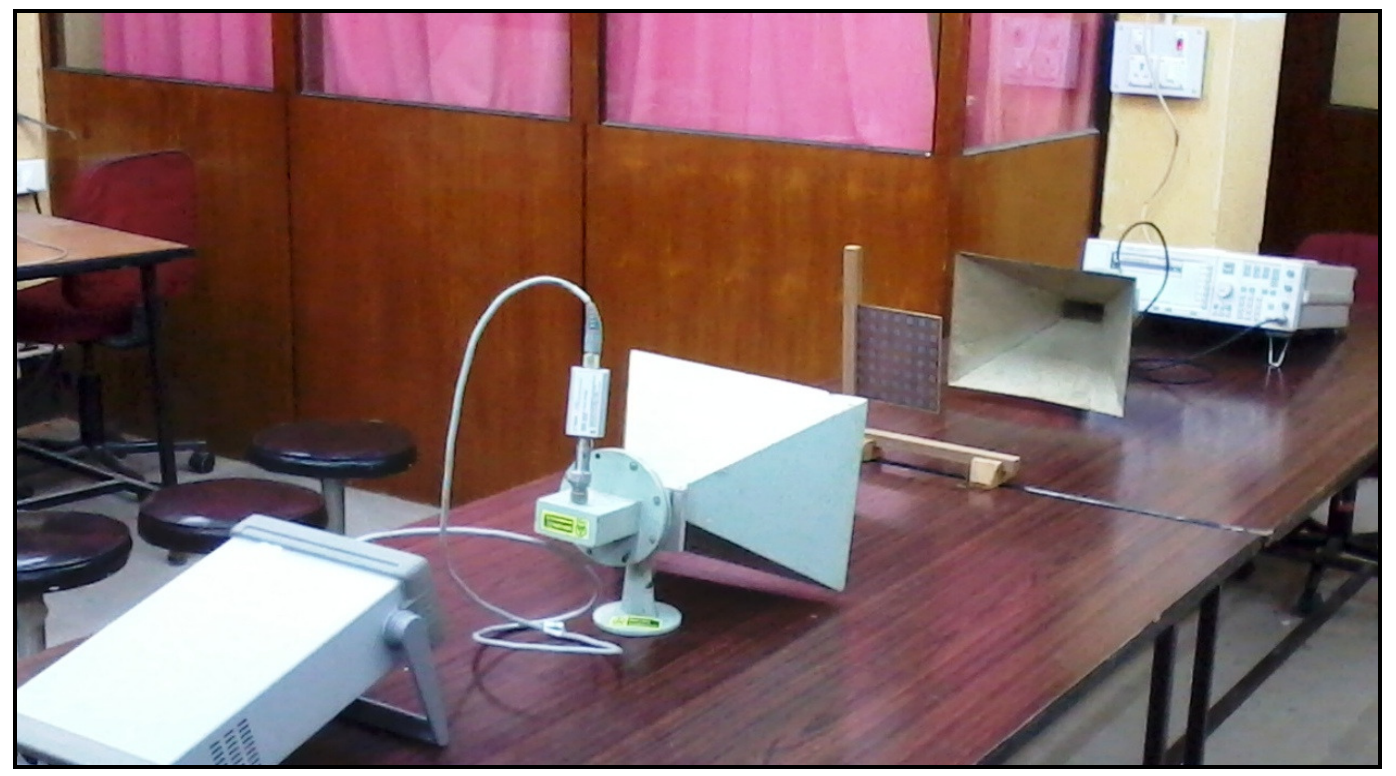

Fig 11. Experimental setup.

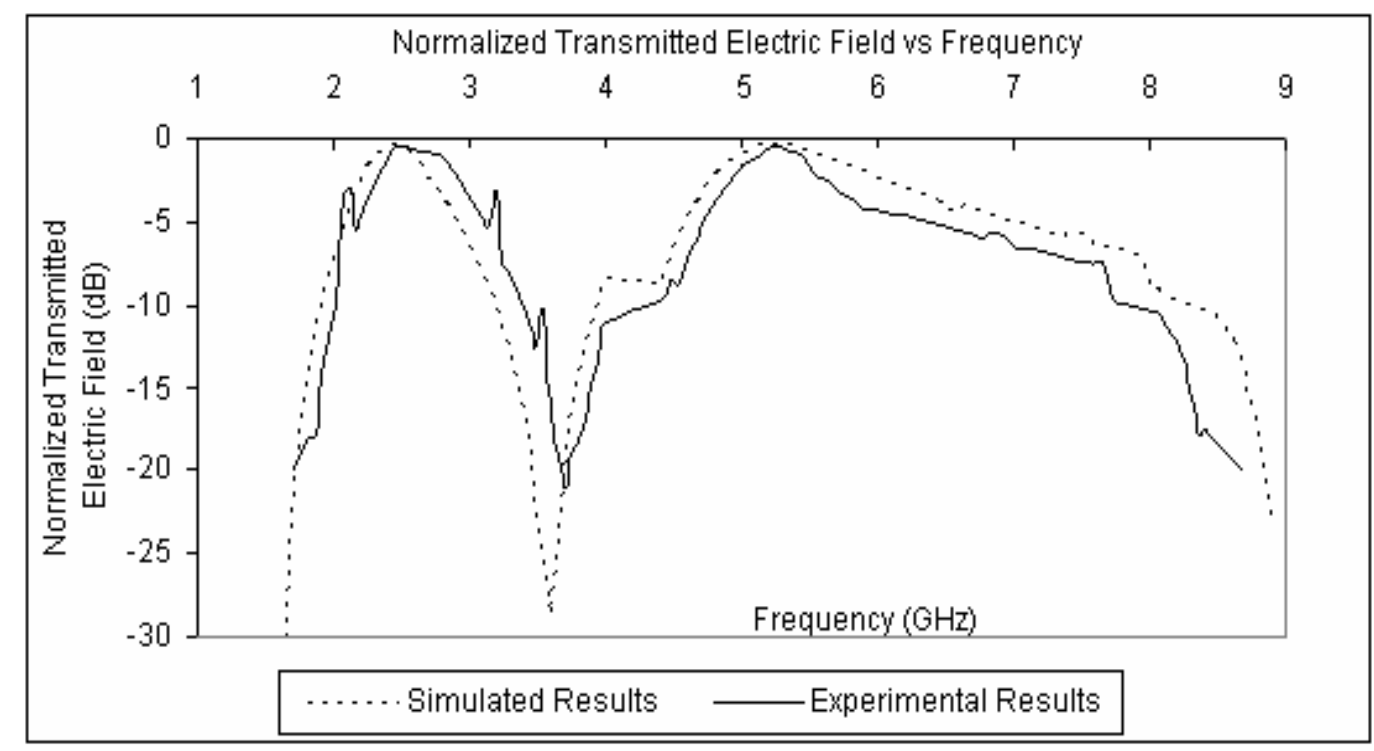

Fig 12. Normalized transmitted electric field vs frequency for FSS shown in Fig 8. 
The equivalent circuit model is shown in Fig. 13, which can be used as the electrical counter part of the designed spatial filter. As discussed above, the loop (shown in Fig. 1 and Fig. 6) is modelled as a serial LC resonant circuit and the complementary (shown in Fig. 2 and Fig. 7) is modelled as a parallel LC resonant circuit, respectively. The substrate between them can be considered as a short transmission line [19], whose length is equal to the thickness of the substrate. The mutual inductance is modelled to represent the coupling effect between the two metallic layers which is equivalent to their far field interactions.

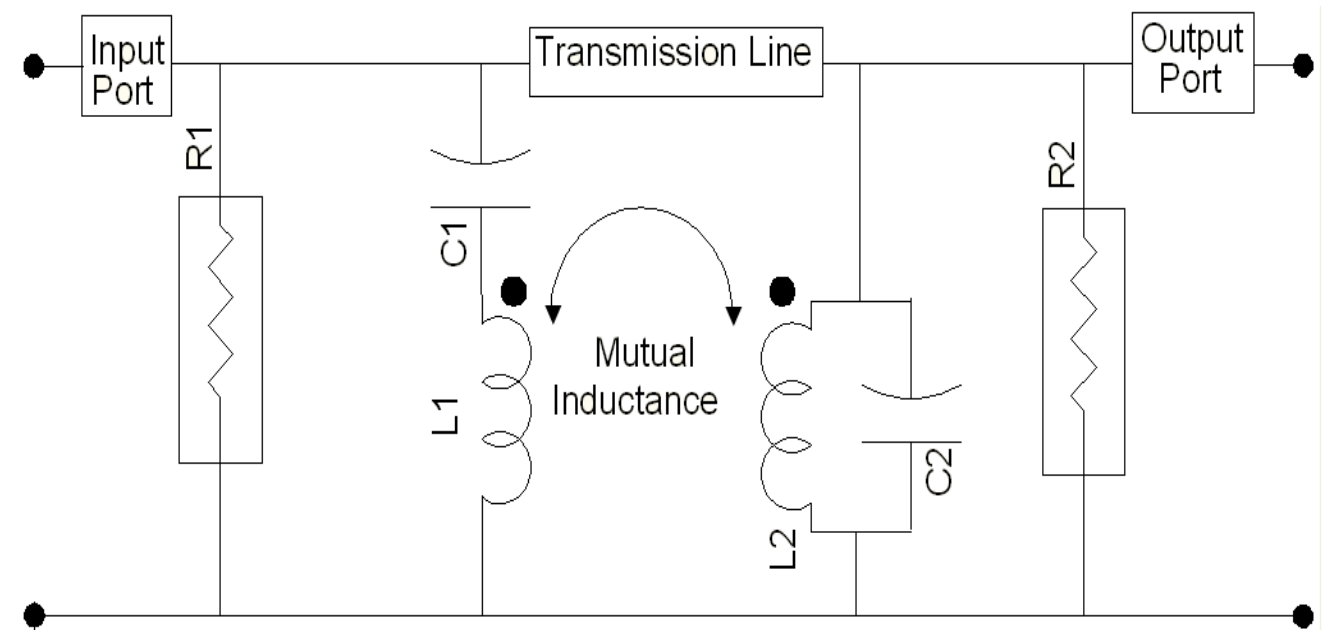

Fig 13. Equivalent circuit model for the designed FSS

The thickness $(\mathrm{t})$ of the PTFE substrate $(0.79 \mathrm{~mm})$ is practically small in our design according to T.K.Wu [15]; so the equivalent electrical length of the transmission line $(\rho)$ is

$$
r=t / l=t \times f / c=\sqrt{e m} \times t \times f
$$

where $\lambda$ is the wavelength, $\mathrm{f}$ is the corresponding frequency, $\mathrm{c}$ is the speed of light, $\varepsilon$ is the permittivity and $\mu$ is the permeability of the medium (the substrate in this case) thus $\rho$ becomes much smaller than one, and this means that the transmission line can be considered as a conductive wire for all practical purpose. Then, the equivalent circuit model can be simplified into a lumped-element model, and its input impedance can be written as

$$
Z_{\text {in }}=\frac{j w L_{1}+\left(1 / j w C_{1}\right)}{-w^{2} L_{1} L_{2}-1 /\left(w^{2} C_{1} C_{2}\right)+L_{1} / C_{2}+L_{2} / C_{1}}
$$

Where $\omega$ is the angular frequency, $\mathrm{j}=\sqrt{ }-1$ and $\mathrm{C}_{1}, \mathrm{C}_{2}, \mathrm{~L}_{1}, \mathrm{~L}_{2}$ are shown in Fig. 13. It can be seen from above equation that the surface impedance has two impedance poles and one impedance zero. When the impedance becomes zero, the circuit current goes straight into the ground, and there is no transmission; however when the impedance goes to infinity, the circuit develops high impedance, and 
the wave is transmitted. This is clearly understood from normalized transmitted electric field vs frequency plots of the FSSs shown in Fig. 4 and Fig. 12.

From Fig. 12 the transmission coefficients show insertion losses of less than $0.4 \mathrm{~dB}$ which is mainly due to the finite thickness of the substrate. Because the relative permittivity of air and substrate are different from each other, a part of the wave energy is lost due to scattering at the surface.

\section{CONCLUSION}

The experimental and simulated theoretical analyses are tabulated in Table I for ease of comprehensive understanding.

TABLE I. SUMMARY OF RESULTS

\begin{tabular}{|c|c|c|c|c|}
\hline \multicolumn{2}{|c|}{ FSS Structure } & $\begin{array}{c}\text { Resonant Frequency } \\
(\mathbf{G H z})\end{array}$ & $\begin{array}{c}\text {-10dB Bandwidth } \\
(\mathrm{GHz})\end{array}$ & $\begin{array}{l}\text { Normalized Transmitted } \\
\text { Electric Field } \\
\text { (dB)/Insertion Loss (dB) }\end{array}$ \\
\hline \multirow[b]{2}{*}{$\begin{array}{c}\text { FSS Structure shown } \\
\text { in Fig } 3\end{array}$} & \multirow[b]{2}{*}{ Simulated } & 2.52 & 1.8 & -0.12 \\
\hline & & 6.91 & 3.6 & -0.32 \\
\hline \multirow{5}{*}{$\begin{array}{c}\text { FSS Structure shown } \\
\text { in Fig } 6\end{array}$} & \multirow[b]{2}{*}{ Simulated } & 2.42 & 1.3 & -0.11 \\
\hline & & 5.22 & 4.0 & -0.22 \\
\hline & \multirow{3}{*}{ Experimental } & 2.40 & 1.5 & -0.21 \\
\hline & & & & \\
\hline & & 5.20 & 3.8 & -0.31 \\
\hline
\end{tabular}

The newly designed FSS structure (shown in Fig. 8) thus acts like a wide bandpass filter transmitting well at WLAN frequencies of $2.4 \mathrm{GHz}$ and $5.2 \mathrm{GHz}$. Considering $-10 \mathrm{~dB}$ as the transmission limit the percentage bandwidths of $62.5 \%$ and $73.01 \%$ respectively are noted at the resonant frequencies which are remarkable. So in this paper we have successfully designed a tuned, optimized, miniaturized element FSS worthy of WLAN applications with a suitable equivalent electrical circuit analogy.

It is also note worthy that due to the symmetrical nature of the design; the FSS is polarization independent for $90^{\circ}$ (degree) rotations about any vertical axis, perpendicular to the FSS plane and passing through its centre.

\section{REFERENCES}

[1] B. A. Munk, Frequency-Selective Surfaces: Theory and Design. New York: Wiley, 2000.

[2] B. Munk. Finite Antenna Arrays and FSS. Wiley \& Sons, New York, 2003.

[3] N.D. Agrawal and W.A. Imbraile, Design of a Dichroic Cassegrain Sub reflector,IEEE Trans, AP- 27 (4), pp. 466473,1979 . 
[4] G.H.-h Sung, K.W Sowerby,. M.J Neve and. M.J Williamson A.G, A Frequency selective Wall for Interface Reduction In Wireless Indoor Environments, Antennas and Propagation Magazine, IEEE, Vol 48, Issue 5, Oct 2006, pp 29-37.

[5] H.Y. Chen and Y. Tao ,Bandwidth Enhancement Of A U- Slot Patch Antenna Using Dual-Band Frequency-Selective Surface With Double Rectangular Ring Elements, Microwave And Optical Technology Letters, Vol. 53, No. 7, July 2011,pp-1547-1553

[6] T. K. Wu. M. Zimmertnan, and S. W. Lee, Evaluation of frequency selective reflector antenna svstems, Microwave and Optical Technol. Lett., vol. 6, no. 3. pp. 175-179, Mar. 1993.

[7] T. K. Wu, Cassini frequency selective surface development, J. Electromagnetic Waves Application., vol. 8, no. 12, pp. 1547-1561, Dec. 1994.

[8] A.L.P.S. Campos, R.H.C. Maniçoba, L.M. Araújo, , A.G d'Assunção. , Analysis of A Simple FSS cascading with Dual-Band Response, IEEE Transactions on Magnetics,Vol. 46, issue. 8, pp. 3345-3348, August 2010.

[9] H. Zhou, S.B. Qu, B. Q. Lin, J.Q. Zhang, C. Gu, H. Ma, Z. Xu, P. Bai, W.D. Peng, Dual band frequency selective surface based on circular aperture-coupled patches, Microwave and Optical Technology Letters, Vol 53, Issue 8 , pp. 1784-1786, August 2011.

[10] L. M. Araujo, R. H. C. Manicoba, A. L. P. S. Campos,and A. G. d'Assuncao , A Simple Dual-Band Frequency Selective Surface, Microwave and Optical Technology Letters, no. 4, pp. 942-944, April. 2009.

[11] X.D. Hu , X.L. Zhou, L. S. Wu, L. Zhou, and W.Y. Yin , A Novel Dual-Band Frequency Selective Surface (FSS), Microwave Conference ,APMC 2009,Asia Pacific , pp. 1227 - 1230 , 2009.

[12] IEEE 802.16 Working Group on Broadband Wireless Access Standards, http://grouper.ieee.org/groups/802/16/index.html

[13] IEEE 802.11 -Wikipedia http://en.wikipedia.org/wiki/IEEE_802.11

[14] Y.T. Lo and S.W. Lee, Antenna handbook, Van Nostrand Reinhold Co., New York, 1988, pp 13.13-13.20.

[15] T.K. Wu, Frequency Selective Surface and grid array (A Wiley Interscience publication), pp. 5-7, 1995

[16] D. M. Pozar, Microwave Engineering, $3^{\text {rd }}$ Edition, Wiley, 2006.

[17] B. Monacelli, J. B. Pryor, B. A. Munk, D. Kotter, and G. D. Boreman, Infrared frequency selective surface based on circuit-analog square loop design, IEEE Trans. Antennas Propag., vol. 53, no. 2, pp. 745-752, Feb. 2005.

[18] D. M. Pozar, Microstrip Antennas, Proc. IEEE, Vol. 80, No. 1, pp. 79-81, January 1992.

[19] K. Sarabandi and N. Behdad, A frequency selective surface with miniaturized elements, IEEE Trans. Antennas Propag., vol. 55, no. 5, pp. 1239-1245, May 2007 